\title{
Biodiversity on the brink
}

\author{
The new United Nations panel can offer real hope of illuminating the threat to biodiversity, not least \\ from climate change.
}

Predicting the scale of biodiversity loss this century from climate change is a formidable challenge. At present we recognize about 2 million species, but estimates of the total number of species on Earth range from about 5.5 million to tens of millions. Despite our incomplete knowledge, we do know however that the promise of world leaders to significantly reduce the rate of global biodiversity loss by 2100 has failed. In 2002, Parties to the Convention on Biological Diversity committed to a significant reduction of the current rate of biodiversity loss at a global, regional and national level.

Yet, at current rates species extinctions could very well outpace new discoveries. The decline in global biodiversity - which has been $30 \%$ since 1970 - continues unabated. In the oceans, overfishing has eroded blue-fin tuna numbers to $18 \%$ of their number just 40 years ago, and on land, deforestation removes millions of hectares of pristine forest habitat each year. A study last year that looked at a host of biodiversity impacts - from extinctions to shifts in distribution and habitat loss for terrestrial, fresh water and marine ecosystems worldwide - predicted that for a range of possible scenarios, biodiversity will continue to decline over the twenty-first century (Science 330, 1496-1501; 2010).

But how much of the ongoing and anticipated loss is attributable to climate change? Recent evidence suggests that one in every species could face extinction by 2100 from climate change alone (Proc. Natl Acad. Sci. USA 108, 12337-12342; 2011). Yet, this startling figure may well be conservative. Although it has been possible to point to anthropogenic climate change as a driver of global biological changes, assessing the extent to which regional changes in biodiversity are caused by greenhouse-gas warming has proven particularly intractable, not least because of the need to disentangle the effects of climate change from those of other drivers such as pollution or overexploitation (Nature Clim. Change 1, 2-4; 2011).

Moreover, the real scale of biodiversity loss due to climate change could be underestimated if it is only examined at the morphospecies level, as research on page 313 suggests. Using mitochondrial DNA variability, Steffen Pauls and colleagues show that loss of genetic diversity will outpace range contractions for nine mountain-dwelling aquatic insect species in Europe as the climate warms. The implication of their research, that analysis of intraspecific genetic diversity is needed to gauge the true extent of biodiversity loss, is backed up by other recent studies in species from the middle spotted woodpecker Dendrocopus medius (Ecography http://dx.doi.org/10.1111/ j.1600-0587.2011.06713.x; 2011) to the neotropical tree Caryocar brasiliense (Tree Genet. Genomes http://dx.doi.org/10.1007/ s11295-011-0409-z; 2011). Collectively, this growing body of research points to the necessity of accounting for genetic diversity in both measuring and conserving biodiversity.

\section{Halting the threat of a sixth extinction must begin in earnest, and cannot wait for greater certainty in our estimates of extant biodiversity and predictions of its fate.}

Although such research suggests that biodiversity loss may be underestimated, the incredibly complex nature of biodiversity let alone its interaction with the climate clearly leaves much room for expanding and advancing the research agenda on ecology and climate change. On this front, ecology is continually revealing new complexities, as highlighted by two articles in this issue. A News and Views on page 300 discusses new research by Dedmer Van de Waal and colleagues,' published in The ISME Journal, that highlights a positive impact of climate change on ecosystems: as concentrations of carbon dioxide in surface waters increase, the toxic forms of the cyanobacteria Microcytis that routinely pose health problems to freshwater ecosystems may decrease in numbers as they are outcompeted by their non-toxic counterparts. Furthermore, in a Letter on page 308, Riccardo Rodolfo-Metalpa and co-authors provide a compelling case that calcifiers subjected to ocean acidification are more resilient when protected by external organic tissue, and that this previously underappreciated morphological attribute can play a role in how marine biodiversity is impacted by climate change.
Undoubtedly, further discoveries of this kind will abound in the future. Meanwhile, halting the threat of a sixth extinction must begin in earnest, and cannot wait for greater certainty in our estimates of extant biodiversity and predictions of its fate. In this regard, the recently launched United Nations panel on biodiversity, which is due to meet formally for the first time next month in Nairobi, Kenya, is to be welcomed. At its October meeting, the panel - known as the Intergovernmental Science-Policy Platform on Biodiversity and Ecosystem Services, or IPBES - will discuss its remit and how it will carry out its proposed work agenda in practice.

Much like the Intergovernmental Panel on Climate Change (IPCC) on which it is modelled, the IPBES will not undertake scientific research, but will assess scientific knowledge with the goal of informing policy. Though its intention is not to steer science, in shining a light on the problem of biodiversity loss and in evaluating its economic ramifications, the IPBES will almost certainly expose the need for ramped up investments in research.

The panel can learn much from the experience of its climatic counterpart - the IPBES will also face the challenge of whether and how to assess the voluminous body of literature that is commissioned and published outside of academia, by conservation and other agenda-driven organizations. Here, a principle of transparency must be adopted rather than one of exclusion (Nature Clim. Change 1, 227; 2011). In considering the logistics of assessing a topic of enormous complexity, the IPBES can look at the pros and cons of the current IPCC set-up. Implementing consistent policies across working groups, as well as methods for communicating results, will be a necessity, as will ensuring that this interdisciplinary subject is approached by a diverse range of experts.

More crucially, however, given the probable scale of climate impacts on biodiversity and the associated uncertainty, climate experts must clearly play a very significant role in the IPBES. The IPBES and the IPCC must therefore find a way of formally collaborating. Together, these expert panels can offer real hope of pointing to the policies needed to get us back on track for reducing the rate of biodiversity decline in a warming world. 